\title{
Nonlinear Dynamics in Directly Modulated Semiconductor Lasers with Optical Loop Mirror Feedback
}

\author{
Hani J. Kbashi, ${ }^{1,2}$ \\ ${ }^{1}$ Department of Physics, College of Science, University of Baghdad, Baghdad, Iraq \\ ${ }^{2}$ School of Physics and Astronomy, University of Southampton, Southampton, UK \\ Email: h.kbashi@soton.ac.uk
}

Received August 7, 2012; revised September 12, 2012; accepted September 27, 2012

\begin{abstract}
The investigation of the nonlinear dynamics of a semiconductor laser based on nonlinear optical loop mirror (NOLM) feedback using Ge doped optical fiber was carried out experimentally. Animations of compilations of the output power as a function of time series and phase plane with effects of optical feedback level, carrier current and modulation signal strength are demonstrated as a tool to give insight into the laser dynamics. Different dynamic states, including $2 \times, 4 \times$ multiplication and quasi-periodic and periodic frequency-locked pulsing states extended to chaotic behaviour were observed by varying the parameters of modulated frequency and optical feedback strength. The frequency-locked pulsing states were observed to exhibit a harmonic frequency-locking phenomenon and the pulsing frequency is locked to a harmonic nonlinearity in loop instead of the modulated frequency.
\end{abstract}

Keywords: Nonlinear Dynamics; Nonlinear Loop Mirror; Pulse Multiplication; Optical Chaotic; Pulse Mode Locking

\section{Introduction}

Nonlinear dynamics of semiconductor lasers have been widely studied due to the important roles semiconductor lasers play in conventional and chaotic optical communication systems. Under external perturbations such as optical feedback [1], optical injection [2], and optoelectronic feedback $[3,4]$, various nonlinear dynamics and routes to chaos have been observed and investigated in semiconductor laser [5]. Optical feedback is performed by re-injecting laser emission in external-cavity or by ring-cavity geometries. Hence, numerous theoretical and experimental studies on dynamical regimes in semiconductor lasers with optical feedback by re-injecting laser emission [6-9] and few numerical studies with ring cavity have been reported [10,11].

The intensity-dependent transmission characteristic of the NOLM is originally based on a nonlinear differential phase shift between the counter-propagating beams in the loop. This intensity is generally attributed to self-phase modulation (SPM) and a variation of the differential phase by $\pi$ will cause a change of the NOLM from high transmission to a high reflection. However, such differential phase shift may appear only if the NOLM power symmetry is broken in some ways. One way to obtain a differential phase is to use an asymmetrical coupler in the NOLM. In such case, the dynamic range and the critical power are directly dependent on the coupling ratio with low insertion. A second possible way is to use a symmetrical coupler in the NOLM and an attenuator in the loop which results in high dynamic range and low critical power, even when the insertion loss is high [12]. When applying feedback to semiconductor laser through NOLM, it behaves as an external optical resonator and can be used to generate bistable and chaotic output [13]. To date the reported nonlinear dynamics have been optoelectronics and optical re-injecting feedback. While, nonlinear dynamic with NOLM has been conventionally modelled using complex Ginzburg-Landau in fiber lasers, such as the figure of eight laser and the nonlinear polarization rotation technique laser [14]. In this paper, we report an experimental demonstration of nonlinear dynamic system of semiconductor laser using a nonlinear optical loop mirror (NOLM) feedback. DC bias current, the modulation frequency, and feedback strength are taken in account as controllable parameters of this nonlinear loop mirror feedback.

\section{Experimental Setup}

The schematic representation experimental setup of nonlinear dynamics based on NOLM feedback is shown in Figure 1. In this NOLM feedback system, an InGaAsP-InP single-mode DFB laser diode with a centre 


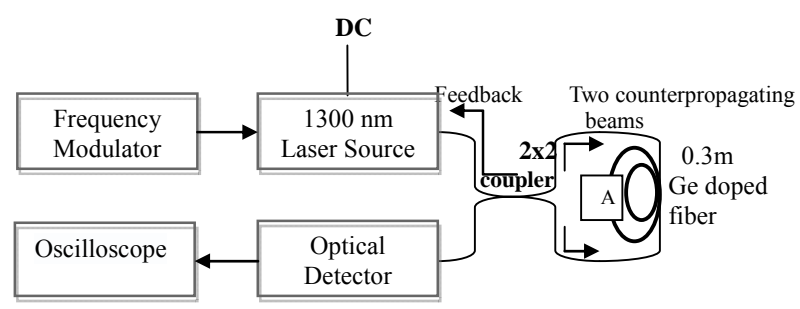

Figure 1. Experimental setup of a nonlinear dynamic based on NOLM.

wavelength at $1300 \mathrm{~nm}$ and threshold is $14 \mathrm{~mA}$ was used. The laser is DC biased with external modulation current source. The output power from the laser is connected to the first input part of $2 \times 2$ optical directional coupler. The two output parts of coupler are connected together through $1 \mathrm{~m}$ loop length and an optical attenuator (A). 30 $\mathrm{cm}$ from this loop is $20 \%$ Ge doped optical fiber with 10 $\mu \mathrm{m}$ core diameter. The Ge doped fiber is located in mid of loop as a nonlinear fiber to modified transmission properties of NOLM and generated high nonlinear dynamic range with low critical power. The reflected light from coupler is split in two ways, one reflected as feedback to the cavity of semiconductor laser and the other is detected and converted into an electrical signal with a fast amplified telecom photodiode. This electrical signal is observed in an oscilloscope. The controllable parameters of this optical feedback system are compose of the DC bias current, modulation current source whose modulated frequency changed, the feedback strength using Ge doped fiber and an attenuator, as shown in Figure 2. The modulation current source provides periodic timing slots to produce a regular pulse train, while nonlinear fiber shortens the pulse compared to that expected from generator. By adjusting these parameters, the system could be operated in different dynamic states.

\section{Results and Discussion}

The characteristics of the states found in various dynamic NOLM feedback systems are plotted for time series on the left-hand side of Figures 2(a)-(e), and the phase portraits on the right-hand side of the figure. From these set of figures, the original periodic pulse, and its phase plane can be observed (Figure 2(a)). With high DC bias (100 $\mathrm{mA})$ and large frequency modulation $(50 \mathrm{MHz}), 2 \times(100$ $\mathrm{MHz})$ and $4 \times(200 \mathrm{MHz})$ multiplications were observed (Figures 2(b) and (c)) respectively due to the competition between the effect of optical feedback and modulation frequency. The multiplication times as well as the loop frequency and loop harmonic are depends on the optical power propagate in loop and frequency modulated. As the loop frequencies are varied under strong modulation and high nonlinearity in loop, various frequency-locked pulsing states with $20 \mathrm{MHz}$ repetition rate were observed (Figures 2(d) and (e)). Here, the pulsing frequency of the laser locks to one of the harmonics of the delay loop frequency instead of the loop frequency itself. Chaotic behaviours as in Figure 2(f) were observed in this setup between pulse multiplications and pulse mode locking. Weak chaotic signal was generated near laser threshold $(14 \mathrm{~mA})$ and strong chaotic signal was generated with strong feedback $(-12 \mathrm{dBm})$ and high modulation frequency $(100 \mathrm{MHz})$.

From the phase plane obtained, the original periodic pulse was stable node and the limit cycle around that vicinity was a stable limit cycle, whereas in $2 \times, 4 \times$ multiplications and chaotic behavior, there is neither stable limit cycle nor stable node on the phase planes even with the ideal multiplication parameters. However, as the multiplication factor increases, the system trajectories moved away from the origin. Corresponding to the modulated frequency and fundamental cavity round-trip time, the laser operating in fundamental quasi-periodic as in (Figure 2(d)) and in a periodic as shown in (Figure 2(e)) mode-locking regime with only a single pulse circulating intracavity. The change from quasi-periodic to periodic mode-locking was due to more adjusting between modulated frequency and nonlinear phase shift generated in $\mathrm{Ge}$ fiber. The vicinity of quasi-periodic mode-locking was not stable cycle because the adjusting between modulated frequency and nonlinearity in Ge doped loop mirror was not high enough to generated uniform periodic modelocking, other hand; higher stability in periodic modelocking was result with more accuracy adjusting. In harmonic frequency locking pulse, the laser output was characterized using autocorrelator pulse duration measurements, as shown in Figure 3. The optimum output characteristics in terms of both shortest pulse duration which is about 1 Psec and highest repetition rate were observed when the modulated frequency was higher [15]. These variations in the laser dynamics was due to the change of the modulated frequency and modulation current and their interplay with the nonlinear phase shift in Ge fiber. The intensity spikes correspond to ringing in the electronic system excited by the photodiode's impulse response to a very short optical pulse.

\section{Conclusion}

In conclusion, the nonlinear dynamics of a semiconductor laser with nonlinear optical loop mirror feedback were investigated experimentally. The combined of modulated frequency and managed effects of the nonlinear give rise to $2 \times, 4 \times$ multiplications, chaotic behaviours and sensitive to the repetition rate mode locking mechanism. This combined action of nonlinear effects inside Ge fiber acts similar to saturable absorber action and improving various features of the dynamic laser, such as increasing the side mode suppression and narrowing the line width. 

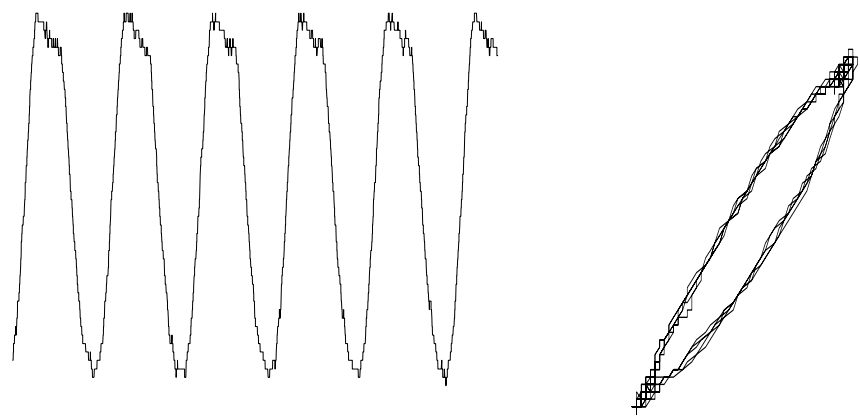

-1.80E-007-1.20E-007-6.00E-0080.00E+000 6.00E-008 1.20E-007 Time (sec)

(a)
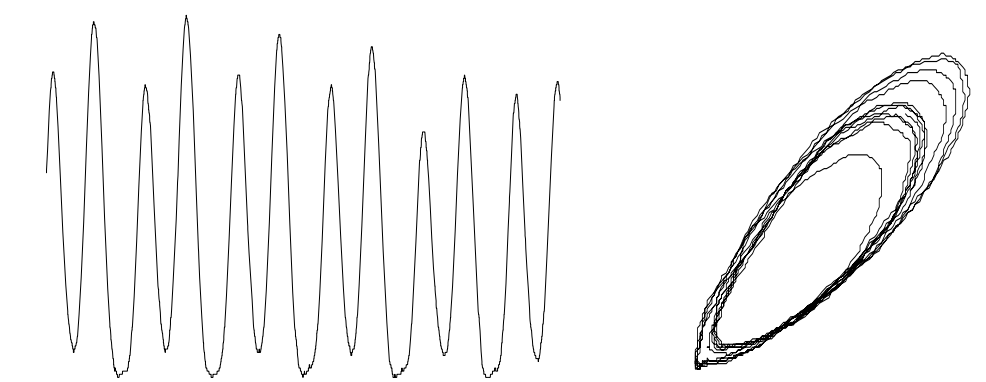

-1.20E-007-6.00E-008 0.00E+000 $6.00 \mathrm{E}-008$ 1.20E-007 Time (sec)

(b)

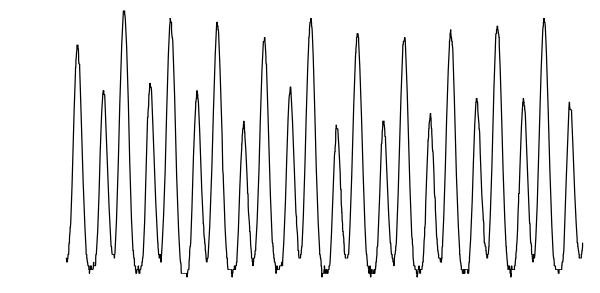

-1.20E-007-6.00E-008 0.00E+000 6.00E-008 1.20E-007 Time (sec)

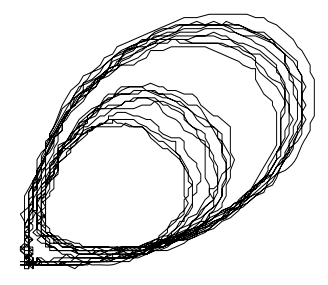

(c)
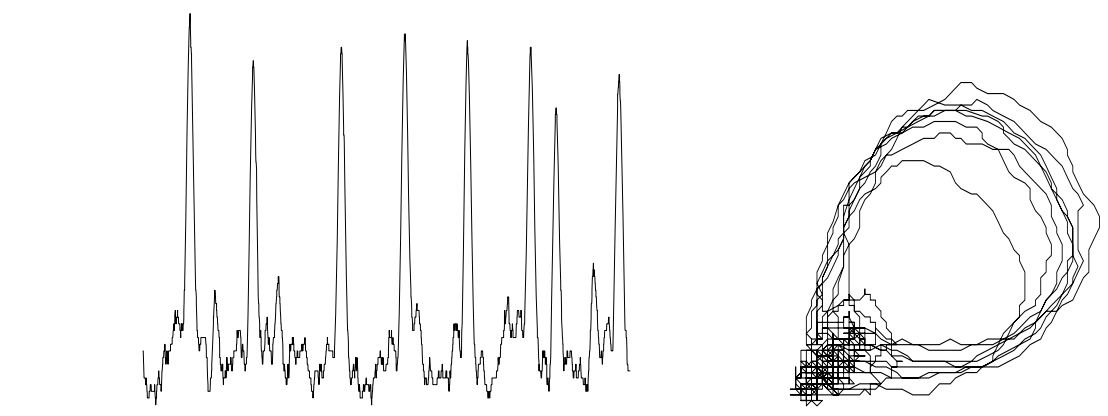

$\begin{array}{llllll}-0.00000030 & -0.00000015 & 0.00000000 & 0.00000015 & 0.00000030\end{array}$ Timme (sec)

(d) 

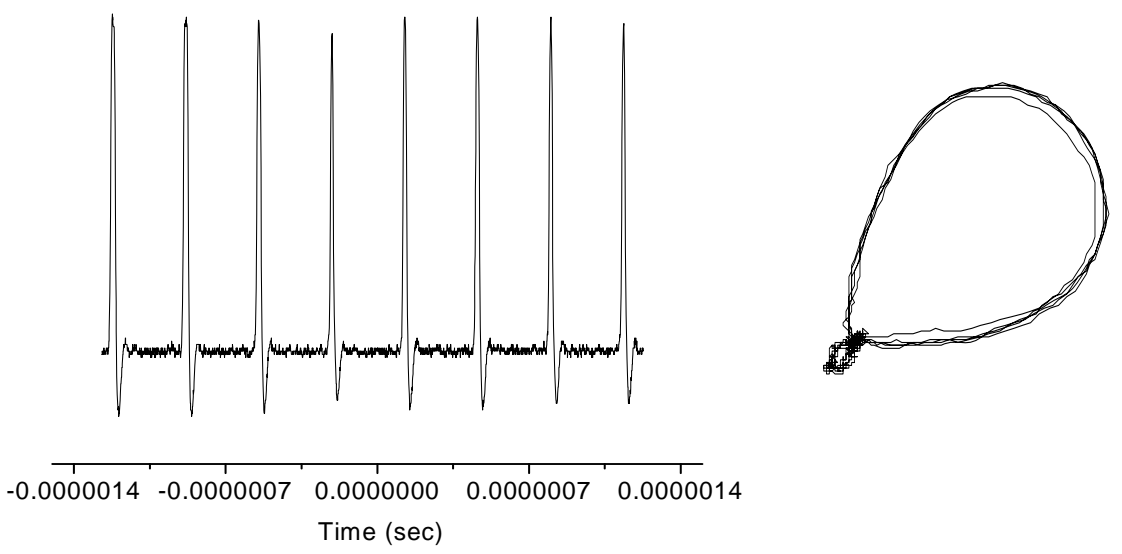

(e)
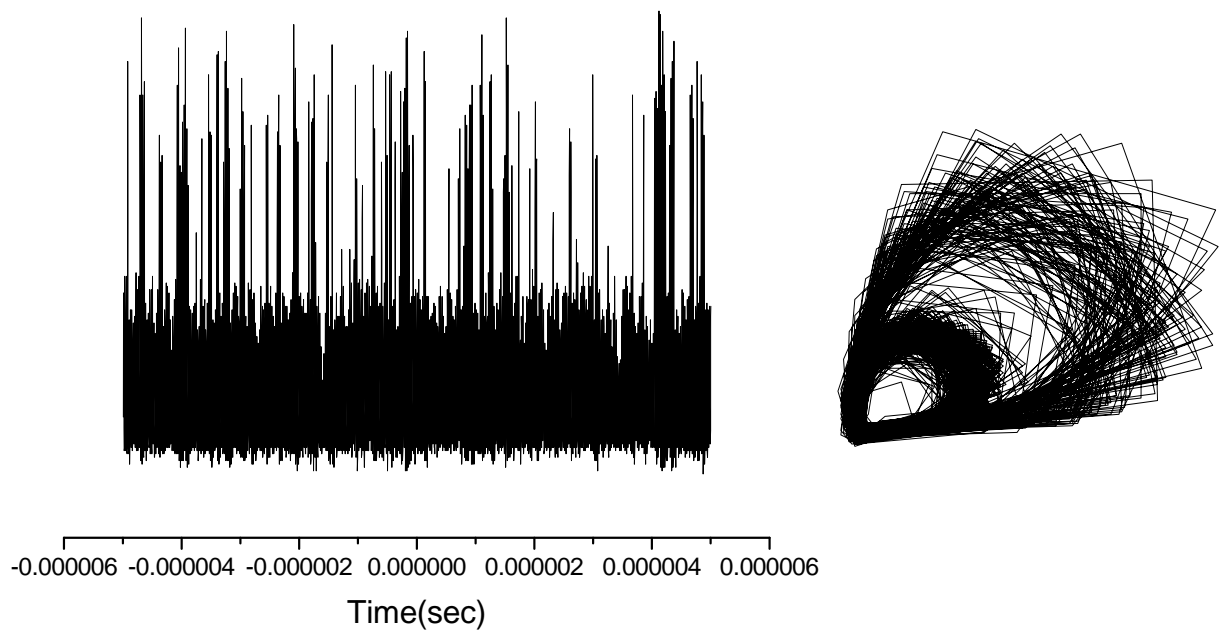

(f)

Figure 2. (Left) Laser output power as a function of time, (Right) phase plane. (a) Original pulse; (b) 2× multiplication; (c) 4× multiplication; (d) Non periodic mode locking; (e) Periodic mode locking and (f) Chaotic behavior.

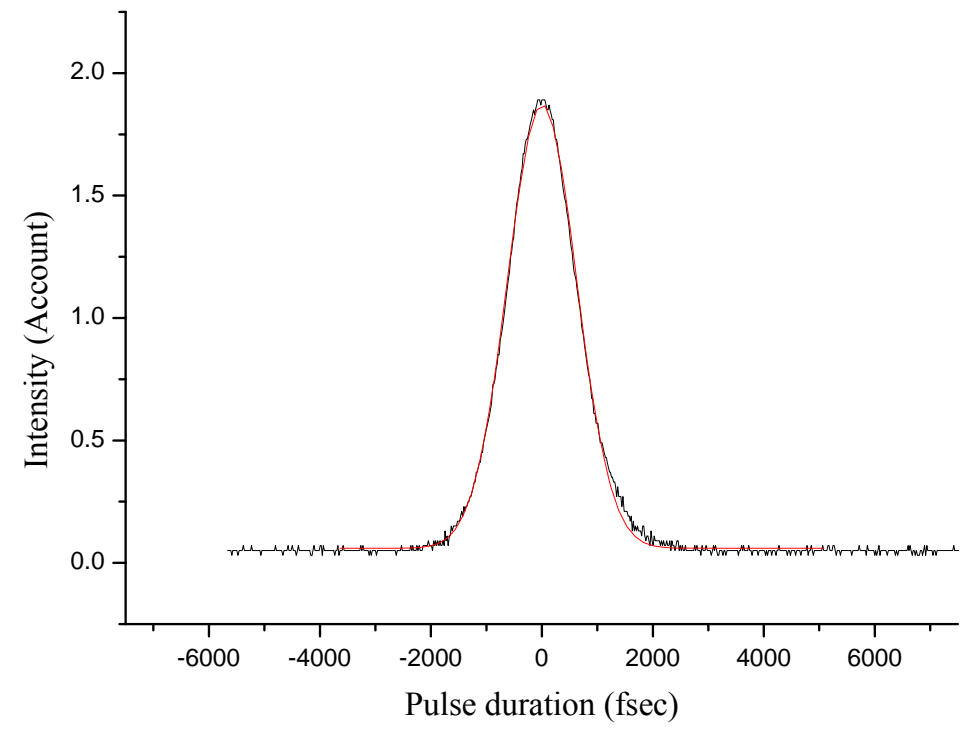

Figure 3. Experimentally recorded autocorrelations laser pulses. The red line shows a fit to the expected hyperbolic secant squared. The full-width of this autocorrelation, obtained from the fit curves is 1 ps. 


\section{Acknowledgements}

This work was supported by both of Institute of International Education/SRF and CARA.

\section{REFERENCES}

[1] J. Blakely, J. N. Illing and L. Gauthier, "High-Speed Chaos in an Optical Feedback System with Flexible Timescales," IEEE Quantum Electronics, Vol. 40, No. 3, 2004, pp. 299-305.

[2] S. Banerjee, P. Saha and A. R. Chowdhury, "Optically Injected Laser System: Characterization of Chaos, Bifurcation, and Control," American Institute of Physics Chaos, Vol. 14, No. 2, 2004, pp. 347-357.

[3] L. Larger and J. M. Dudley, "Optoelectronic Chaos," Nature, Vol. 465, 2010, pp. 41-42.

[4] H. J. Kbashi, "RF Chaos Communication System Based on Optoelectronic Feedback," Optoelectronics and Advanced Materials-Rapid Communications Vol. 4, No. 8, 2010, pp. 1091-1094.

[5] F. Lin and J. Liu, "Nonlinear Dynamics of a Semiconductor Laser with Delayed Negative Optoelectronic Feedback," IEEE Journal of Quantum Electronics, Vol. 39, No. 4, 2003, pp. 562-568.

[6] M. Lee, J. Paul, I. Pierce and K. A. Shore, "Frequency-Detuned Synchronization Switching in Chaotic DFB Laser Diodes," IEEE Journal of Quantum Electronics, Vol. 41, No. 3, 2005, pp. 302-307.

[7] X. F. Li, W. Pan, B. Luo, D. Ma, N. H. Li and Y. Wang, "Suppressing Nonlinear Dynamics Induced by External Optical Feedback in Vertical-Cavity Surface-Emitting Lasers," Optics and Laser Technology, Vol. 37, No. 6, 2005, pp. 438-443.

[8] J. P. Toomey, D. M. Kane, M. W. Lee and K. A. Shore, "Nonlinear Dynamics of Semiconductor Lasers with Feed- back And Modulation," Optics Express, Vol. 18, No. 16, 2010, pp. 16955-16972. doi:10.1364/OE.18.016955

[9] S. Bauer, O. Brox, J. Kreissl, B. Sartorius, M. Radziunas, J. Sieber, H.-J. Wu"nsche and F. Henneberger, "Nonlinear Dynamics of Semiconductor Lasers with Active Optical Feedback," Physical Review E, Vol. 69, No. 1, 2004, Article ID: 016206. doi:10.1103/PhysRevE.69.016206

[10] K. Spaulding, D. Yong, A. Kim and J. Kutz, "Nonlinear Dynamics of Mode-Locking Optical Fiber Ring Lasers," Journal of the Optical Society of America B, Vol. 19, No. 5, 2002, pp. 1045-1054. doi:10.1364/JOSAB.19.001045

[11] Q. Xu and M. Yao, "Theoretical Analyses on Short-Term Stability of Semiconductor Fiber Ring Lasers," IEEE Journal of Quantum Electronics, Vol. 39, No. 10, 2003, pp. 1260-1265.

[12] B. Ibarra-Escamilla, E. A. Kuzin, P. Zaca-Moran, R. Grajales-Couti, F. Mendez-Martinez, O. Pottiez, R. RojasLaguna and J. W. Haus, "Experimental Investigation of the Nonlinear Optical Loop Mirror with Twisted Fiber and Birefringence Bias," Optics Express, Vol. 13, No. 26, 2005, pp. 10760-10767. doi:10.1364/OPEX.13.010760

[13] C. A. Merchant and A. L. Steele, "A Tunable Nonlinear Optical Loop Mirror with Feedback Using Linear Highly Birefringent Fiber in the Loop," Optics Communications, Vol. 258, 2006, pp. 288-294. doi:10.1016/j.optcom.2005.08.004

[14] A. Karar, T. Smy and A. L. Steele, "Nonlinear Dynamics of a Passively Mode-Locked Fiber Laser Containing a Long-Period Fiber Grating," IEEE Journal of Quantum Electronics, Vol. 44, No. 3, 2008, pp. 254-261, 288-294.

[15] H. J. Kbashi, "Additive Mode Locking Based on a Nonlinear Loop Mirror Ring Laser," Quantum Electronics, Vol. 42, No. 3, 2012, pp. 216-219. doi:10.1070/QE2012v042n03ABEH014707 